ARTÍCULO DE REVISIÓN

\title{
Consideraciones en el tratamiento anestésico en pacientes sometidos a tiroidectomía transoral.
}

\author{
Anesthetic Considerations for treatment in patients undergoing thyroidec- \\ tomy transoral.
}

Secundino González Pardoํㅜ, Silvana Paspuel Yar², Adonis Frómeta Guerra ${ }^{3}$, Sandra Amalia Sánchez Figueredo ${ }^{4}$

DOI. 10.21931/RB/2017.02.02.9

\section{RESUMEN}

La anestesia para la tiroidectomía transoral en adultos inicia con la evaluación preoperatoria del estado de la glándula tiroidea, la repercusión local de la patología tiroidea sobre la vía aérea y los riesgos relacionados con las características del paciente. La cirugía de la glándula tiroidea no se considera una urgencia quirúrgica, por lo que es necesario lograr un estado eutiroideo antes de la cirugía. Para evitar la crisis tirotóxica por un lado se bloquea la síntesis hormonal con antitiroideos de síntesis y por otro se disminuyen los efectos centrales y periféricos de las hormonas tiroideas con betabloqueadores. La técnica de elección es la anestesia general balanceada o total intravenosa. La atención postoperatoria se debe esforzar en investigar la posible presencia de las complicaciones hemorrágicas que pueden poner en riesgo el pronóstico vital y las lesiones de los elementos en contacto con la glándula, como las glándulas paratiroideas y los nervios recurrentes. Los cuidados anestésicos del paciente sometido a tiroidectomía transoral no difieren de las otras técnicas quirúrgicas mínimamente invasiva del tiroides.

Palabras Claves: anestesia, anestesia general balanceda, anestesia total intravenosa, tiroidectomía transoral.

\section{ABSTRACT}

Anesthesia for transoral thyroidectomy in adults begins with preoperative evaluation of the thyroid gland status, local repercussion of thyroid pathology on the airway, and risks related to patient characteristics. Surgery of the thyroid gland is not considered a surgical emergency, so it is necessary to achieve a euthyroid state before surgery. To avoid the thyrotoxic crisis, on the one hand, hormonal synthesis is blocked with synthetic antithyroid drugs and on the other, the central and peripheral effects of thyroid hormones with beta-blockers are reduced. The technique of choice is balanced general or intravenous total anesthesia. Postoperative care should be sought to investigate the possible presence of hemorrhagic complications that may compromise the vital prognosis and lesions of the elements in contact with the gland, such as the parathyroid glands and recurrent nerves. Anesthesia care of the patient undergoing transoral thyroidectomy does not differ from the other minimally invasive thyroid surgical techniques.

Key Words: anesthesia, balanced general anesthesia, total intravenous anesthesia, transoral thyroidectomy.

\section{Introducción}

Con el desarrollo de la medicina mínimamente invasiva se han diseñado múltiples abordajes endoscópicos en diferentes sitios del cuerpo. El área de cabeza y cuello no ha quedado excluida de esta tendencia mundial y en 1996 Gagner describió una paratiroidectomía subtotal endoscópica con insuflación de dióxido de carbono usando 4 trocares cervicales. Seguidamente, Huscher et al. publicaron una técnica similar para la tiroidectomía endoscópica ${ }^{1}$.

Cada vez son más novedosas las técnicas quirúrgicas para abordar el territorio de cabeza y cuello hasta llegar al primer sistema de cirugía robótica de uso clínico generalizado, el $d a$ Vinci, comercializado a finales de $1999^{2}$. Sin embargo, son pocos los estudios publicados sobre el manejo anestésico de los pacientes sometidos a este tipo cirugía, donde se necesita una gran fusión entre el cirujano y anestesista basado en el espacio reducido para ambos poder realizar su trabajo.

El manejo anestésico-quirúrgico para procedimientos otorrinolaringológicos y de cirugía de cabeza y cuello, requiere del conocimiento anatómico, fisiológico y fisiopatológico de los estados mórbidos, en pacientes con distintos rangos de edad, desde la infancia hasta la senectud en diversos escenarios clínicos, que ponen a prueba la creatividad y habilidades de los médicos en este campo, ya que los procedimientos diagnósticos o terapéuticos se desarrollan en regiones muy inervadas como ojos, nariz, boca, faringe, laringe, tráquea y pulmones; que aunado a la rica vascularidad de la cabeza y cuello, con la probabilidad alta de sangrado, repercuten la perfusión del cerebro 3 .

Esta revisión muestra las principales formas de tratamiento anestésico en pacientes sometidos a cirugía mínimamente invasiva de cuello haciendo énfasis en la tiroidectomía transoral.

\footnotetext{
${ }^{1}$ Médico tratante en Anestesiología y Reanimación del Instituto Ecuatoriano de Seguridad Social. Profesor de la universidad técnica del norte. Facultada de Ciencia de la Salud.

${ }^{2}$ Lic. en Hospital San Vicente de Paul. Ibarra Ecuador, Profesor de la universidad técnica del norte. Facultada de Ciencia de la Salud.

${ }^{3}$ Médico tratante de Medicina Interna del Instituto Ecuatoriano de Seguridad Social. Profesor asistente

${ }^{4}$ Médico tratante de Medicina Intensiva del Instituto Ecuatoriano de Seguridad Social. Profesor asistente
}

Correspondencia: secun49@gmail.com 


\section{DESARROLLO}

Los cuidados anestésicos de la cirugía de tiroides no comienzan en el salón de operaciones, sino desde la elección del paciente, lo dividiremos en tres etapas fundamentales:

\section{Preoperatorio. Preparación para la cirugía de la glándula tiroides.}

El manejo anestésico de la tiroidectomía está condicionado por la patología tiroidea y por las posibles complicaciones derivadas de la situación anatómica de la glándula. El tratamiento previo a la cirugía se efectúa según distintos esquemas, dependientes del equipo médico-quirúrgico, del paciente y los recursos disponibles. Sólo en el bocio con hipertiroidismo es necesario el tratamiento preoperatorio para conseguir el estado eutiroideo y evitar la crisis tirotóxica postoperatoria. La finalidad de la preparación es doble, por un lado, se bloquea la síntesis hormonal con antitiroideos de síntesis y por otro se disminuyen los efectos centrales y periféricos de las hormonas tiroideas con betabloqueadores, que además de suprimir algunos signos de tirotoxicosis (hiperexcitabilidad muscular, alteraciones cardiovasculares y de la termorregulación), inhiben la conversión de T4 en T3, reducen la taquicardia, las palpitaciones y el temblor. Si los betabloqueantes están contraindicados, pueden utilizarse alternativas como los antagonistas del calcio, que ejercen acciones similares al propranolol pero por distintos mecanismos. El tratamiento debe iniciarse 7- 14 días antes de la cirugía para lograr el eutiroidis$\mathrm{mo}^{4,5}$. Además de los niveles hormonales de tiroides, se dan gran énfasis a la evaluación de cualquier manejo de vía aérea difícil ${ }^{6}$.

El estudio preanestésico previo a una tiroidectomía responde a los criterios habituales de evaluación del riesgo anestésico, como la decisión de una premedicación ansiolítica, de información al paciente y de preparación para la intervención quirúrgica. En el plano biológico, la cervicotomía es una cirugía programada con un riesgo hemorrágico limitado y que sólo necesita un estudio de hemostasia cuando existen signos de una coagulopatía clínica ${ }^{5}$. En la consulta se deberán evaluar también las anomalías de la función tiroidea y la repercusión sobre la vía aérea de la enfermedad tiroidea ${ }^{5}$.

\section{Valoración de la vía área}

La valoración de la vía aérea se sustenta en la búsqueda de los criterios de intubación difícil: clasificación de Mallampati (Figura 1), movilidad de la columna cervical, implantación de los dientes, distancia tiromentoniana, morfología mandibular, aber- tura bucal e índice de masa corporal ${ }^{5,7,8}$. También se debe evaluar la extensión y la repercusión local del bocio. La disnea, la disfagia y la disfonía son signos clásicos de compresión. No obstante, la presencia de un bocio, incluso voluminoso, no parece aumentar la incidencia de las intubaciones difíciles ${ }^{5,8}$.

La mayoría de los autores, entre ellos Garot ${ }^{5}$ y colaboradores, plantean que las dificultades para la intubación (figura 2) aumentan cuando el bocio es de origen neoplásico. Cuando se asocia a disnea y a una desviación o una compresión de la tráquea, el estrechamiento de la luz traqueal suele ser superior al $30 \%$, lo que en presencia de una limitación de la abertura bucal $(<4,4 \mathrm{~cm})$ hace que la intubación endotraqueal sea más peligrosa.

Una disminución aislada de más del 30\% de la luz traqueal no suele dificultar el proceder. Un profundo conocimiento de la anatomía quirúrgica y la vía del nervio es necesario para proteger de lesiones el paso del tubo endotraqueal. No obstante, el orificio glótico puede estar ascendido o con una desviación lateral9.

El análisis morfológico de un bocio descendido intratorácico y de su repercusión sobre los elementos mediastínicos como la tráquea y los vasos se realiza idealmente mediante una tomografía computarizada (TC) cervicotorácica y/o resonancia magnética $(\mathrm{RM})^{5,10,11}$. La laringoscopia preoperatoria permite evaluar la integridad de las cuerdas vocales. Numerosos equipos recomiendan este examen ${ }^{12}$. En una serie de 761 pacientes con lesión laríngea tras tiroidectomía, el $20 \%$ presentaba una anomalía en la movilidad de las cuerdas vocales en el preoperatorio ${ }^{13}$. Otros equipos realizan la laringoscopia en caso de disfonía con el riesgo de pasar por alto una lesión laríngea oculta ${ }^{14} \mathrm{y} / \mathrm{o}$ de reintervención quirúrgica.

La constatación preoperatoria de la movilidad de las cuerdas vocales, bien por fibrolaringoscopia, bien por laringoscopia indirecta, puede ayudar a decidir la estrategia quirúrgica ${ }^{5}$. Aunque todos los autores reconocen su utilidad, suelen admitir que no la realizan rutinariamente.

En nuestro criterio solo en las situaciones que mencionaremos a continuación es necesario realizar la laringospia: a. En pacientes con antecedentes de cirugía tiroidea o cervical, b. Si el paciente presenta disfonía o cambios en el tono de voz, c. Que vaya a realizarse neuromonitorización intraoperatoria, d. En casos de afección maligna, en casos de cánceres avanzados o anaplásicos se debe complementar con broncoscopia y esofagoscopia, e. En enfermedad benigna, cuando se prevea un mayor riesgo recurrencial, como en grandes bocios endotorácicos con compresión o desplazamiento traqueal.

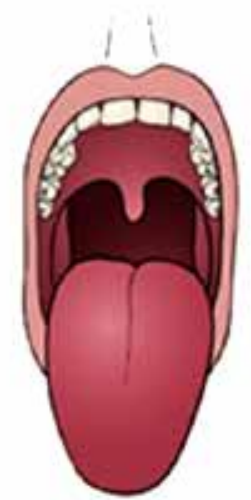

Clase I

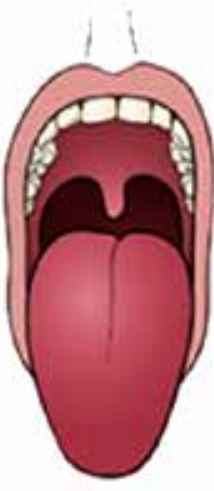

Clase II

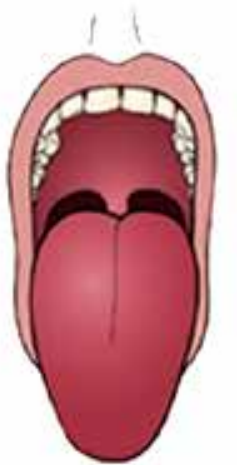

Clase III

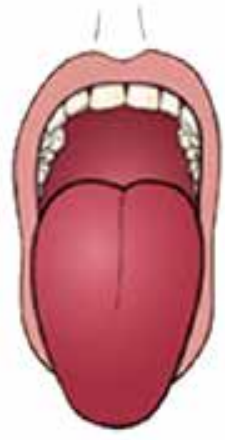

Clase IV

Figura 1: Test de Mallampati: Técnica: paciente en posición sentada, con la cabeza en extensión completa, efectuando fonación y con la lengua fuera de la boca. Indica el tamaño de la lengua y en que magnitud ésta obscurece la visión de la buco-faringe. Clase I: visibilidad del paladar blando, úvula y pilares amigdalinos. Clase II: visibilidad de paladar blando y úvula. Clase III: visibilidad del paladar blando y base de la úvula. Clase IV: imposibilidad para ver paladar blando. Las clases III y IV corresponden a laringoscopia e intubación difícil. 

se despega del plano de la mesa; se han descrito casos excepcionales de compresión medular. Los brazos se mantienen a lo largo del cuerpo, mientras que la mesa se coloca en posición inclinada unos $25^{\circ}$ para favorecer el drenaje venoso cervical. Debido a la falta de acceso a la cabeza, se debe colocar un prolongador para poder realizar las inyecciones fuera del campo quirúrgico. Se coloca una sonda nasogástrica en caso de bocio endotorácico y en algunas ocasiones si se va a realizar un vaciamiento ganglionar, de esta forma se le permite al cirujano una identificación más fácil del esófago ${ }^{5,16,17}$.

La tiroidectomía transoral requiere de un espacio o cavidad para el abordaje con insuflación. El límite máximo de la presión de $\mathrm{CO}_{2}$ debe estar en $10 \mathrm{~mm}$, ya que el objetivo es realizar una pequeña cavidad donde pueda practicarse una disección mínima con los instrumentos que permita la identificación, pinzamiento y extracción. Entre las complicaciones referidas con la utilización de gas tenemos: hipercapnia, acidosis respiratoria y enfisema subcutáneo ${ }^{18}$.

\section{Anestesia general}

Hoy van a cirugía pacientes con patologías complejas que requieren de equipos quirúrgicos especializados (microscopios, equipos endoscópicos, rayo láser, etc.); cerca de $90 \%$ de pacientes necesitan control estricto de la vía aérea (intubación traqueal), por lo tanto, requieren de anestesia general: Anestesia General Balanceada (AGB) o Total Intravenosa Anestesia (TIVA).

En el caso de la cirugía de tiroides transoral se recomienda el uso de cualquiera de las dos variantes con intubación nasotraqueal para el confort del cirujano.

Anestesiología y cirugía evolucionan, para ajustarse a las necesidades individuales del paciente, con base en la investigación de nuevos fármacos anestésicos, con perfiles farmacológicos que ofrecen mayor predictibilidad y seguridad; desarrollando dispositivos más precisos para su administración (vaporizadores y bombas perfusoras TCI guiadas a modo plasma o sitio efecto, basadas en modelos farmacocinéticos-farmacodinámicos); invención de dispositivos específicos para el manejo de la vía aérea; favoreciendo positivamente los resultados. Los agentes anestésicos para la inducción y mantenimiento se eligen de forma individualizada ${ }^{5}$.
Con base en lo anterior exponemos, las recomendaciones generales para las dosis de carga y perfusiones de los opioides empleados comúnmente en la anestesia general para cirugía de tiroides transoral que no difiere en gran medida de las otras vías de abordaje mínimamente invasiva: quedando a cargo del anestesiólogo considerar la variabilidad interindividual; sus covariables, los dispositivos de administración, el estímulo nociceptivo y los retos de cada tipo de cirugía, incluso la pericia, habilidad y experiencia del cirujano en turno ${ }^{3}$.

La inducción de la anestesia general se recomienda por vía IV que brinda mayor biodisponibilidad de fármacos. Se recomienda utilizar ansiolíticos y parasimpaticolíticos, previo a opioides, para reducir la ansiedad, brindar protección neurovegetativa y efecto antisialogogo. Hipnosis (propofol, tiopental, etomidato o midazolam) y BNM (rocuronio, cisatracurio o succinilcolina) para facilitar la ventilación por mascarilla y la instrumentación de la vía aérea. El mantenimiento: de AGB con oxígeno, halogenados (isoflurano, sevoflurano o desflurano) y opioides (bolos múltiples o perfusión) o TIVA: manual (MCI) o infusión controlada por objetivo (TCI), son opciones adecuadas ${ }^{16}$.

Los pacientes quirúrgicos en este tipo de cirugía reciben también infiltración de anestésicos locales con vasopresores (adrenalina) para disminuir sangrado; sin embargo, en combinación con halogenados, aumenta la sensibilidad del miocardio a las catecolaminas exógenas y puede desencadenar arritmias peligrosas para la vida. La cantidad de adrenalina capaz de producir arritmias es: $2.1 \mu \mathrm{g} / \mathrm{kg}$ halotano, $6.7 \mu \mathrm{g} / \mathrm{kg}$ isoflurano, desflurano y sevoflurano, y $10.9 \mu \mathrm{g} / \mathrm{kg}$ enflurano ${ }^{3,16}$.

Los opioides proporcionan excelente analgesia transoperatoria y postoperatoria (excepto remifentanyl). En TIVA se recomienda propofol que posee efectos antieméticos, se debe titular la perfusión manual a tasas entre $90-180 \mu \mathrm{g} / \mathrm{kg} / \mathrm{min}$ (Cp aprox. 3-6 $\mu \mathrm{g} / \mathrm{mL}$ ) o TCI con modelos para pacientes adultos jóvenes y viejos, a modo plasma o sitio efector con dianas entre 3-6 $\mu \mathrm{g} / \mathrm{mL}^{3}$.

En la Tabla 1 representamos los opioides más usados en la práctica médica con sus diferentes características farmacocinéticas y farmacodinámicas.

La inducción con fentanilo se recomienda en bolo manual en pacientes ASA I y II de 3-5 $\mu \mathrm{g} / \mathrm{kg}$ de peso real; pacientes hemo-

\begin{tabular}{|c|c|c|c|c|}
\hline & Fentanilo & Sufentanilo & Alfentanilo & Remifentanilo \\
\hline $\mathrm{pKa}$ & 8,4 & 8 & 6,5 & 7,1 \\
\hline$\%$ no ionizado a $\mathrm{pH} 7.4$ & $<? ?$ & 20 & 90 & ¿67? \\
\hline $\begin{array}{l}\text { Coeficiente de partición } \\
\text { octanol }-\mathrm{H}_{2} \mathrm{O}\end{array}$ & 813 & 1,778 & 145 & 17,9 \\
\hline $\begin{array}{l}\% \text { unidos a proteinas } \\
\text { plasmáticas }\end{array}$ & 84 & 93 & 92 & ¿80? \\
\hline Fracción que difunde (\%) & 1,5 & 1,6 & 8 & ¿13,3? \\
\hline$t^{1} / 2 ?$ (min) & $1-2$ & $1-2$ & $1-3$ & $0,5-1,5$ \\
\hline$t^{1 / 2} 2 ?(\min )$ & $10-30$ & $15-20$ & $4-17$ & $5-8$ \\
\hline$t^{1 / 2} ?(b)$ & $2-4$ & $2-3$ & $1-2$ & $0,7-1,2$ \\
\hline $\mathrm{Vd}_{6}(1 / \mathrm{Kg})$ & $0,4-1$ & 0,2 & $0,1-0,3$ & $0,06-0,08$ \\
\hline $\mathrm{Vd}_{\mathrm{ese}}(1 / \mathrm{Kg})$ & $3-5$ & $2,5-3$ & $0,4-1$ & $0,2-0,3$ \\
\hline Aclaramiento $(\mathrm{ml} / \mathrm{min} / \mathrm{Kg})$ & $10-20$ & $10-15$ & $4-9$ & $30-40$ \\
\hline Razón de extracción hepática & $0,8-1$ & $0,7-0,9$ & $0,3-0,5$ & $\mathrm{NA}$ \\
\hline
\end{tabular}

Adaptado de Bailey PL, Egan TD, Stanley TH: Intravenous opioid anesthetics. In Miller RD, editor: Anesthesia, ed 7. Philadelphia, 2010, Churchill Livingstone, p 791. NA, no aplicable; $\mathrm{pKa}$, constante de disociación iónica; $\mathrm{t}^{1 / 2 \mathrm{a}}, \mathrm{t}^{1} \mathrm{2} \mathrm{b}$, $\mathrm{t}^{1} \frac{1}{2} \mathrm{~g}$, semividas en el modelo tricompartimental; Vdc, volumen de distribución en el compartimento central; Vdee, volumen de distribución en el estado estacionario.

Tabla 1: Datos Físico - Químicos y farmacocinéticos de los agonistas opiodeos que se emplean habitualmente 
dinámicamente estables ASA III, titular 3-4 $\mu \mathrm{g} / \mathrm{kg}$. Es recomendable dar el bolo lento (más de 90 segundos); a mayor velocidad, más efectos secundarios (tórax leñoso y depresión respiratoria) principalmente pacientes en los extremos de la vida. Mantenimiento: fentanyl manual en tasas de perfusión 0.013-0.072 $\mu \mathrm{g} /$ $\mathrm{kg} / \mathrm{min}$ (Cp aprox. 0.001-0.006 $\mu \mathrm{g} / \mathrm{mL}$ ) de acuerdo con el estímulo nociceptivo de cada cirugía, aconsejando disminuir las tasas de perfusión en 30-60 minutos, de 0.052-0.026 $\mu \mathrm{g} / \mathrm{kg} / \mathrm{min}(\mathrm{Cp}$ aprox. 0.004-0.002 $\mu \mathrm{g} / \mathrm{mL}$ ), es decir, cerca del piso de la ventana terapéutica para permitir la ventilación espontánea del paciente y la emersión anestésica entre 10-15 minutos después del cierre de la perfusión ${ }^{3,17}$.

Si la perfusión se prolongara más de 120 minutos, la acumulación es inevitable (50 minutos VMSC) y 180 minutos (100 minutos VMSC), prolongando el despertar. En TCI plasma o sitio efector (dependiendo el modelo FC/FD elegido Scott, Shafer): se sugiere «dianas» de 3-5 ng/mL en pacientes hemodinámicamente estables; el TCI calcula la dosis de carga y la perfusión sobre el tiempo para alcanzar y mantener las Cp y Ce predichas por el modelo utilizado, así como la eliminación y acumulación del fármaco para evitar la sobredosificación (porcentaje decremental de la Cp 20, 50 y 80\%) y de esta forma favorecer la emersión anestésica adecuada sin depresión ventilatoria (Cp aprox. $2 \mathrm{ng} / \mathrm{mL}$ ) y con analgesia residual, principalmente cuando se acompaña de monitoreo cerebral para los componentes del estado anestésico ${ }^{3,16}$.

En la inducción el sufentanilo se recomienda con bolo de 0.3-0.5 $\mu \mathrm{g} / \mathrm{kg}$ equivalentes (Cp aprox. 200-400 pcg/mL), sufentanilo $0.3 \mathrm{ng} / \mathrm{mL}$ combinado con propofol $3.5 \mu \mathrm{g} / \mathrm{mL}$ fueron suficientes para bloquear la respuesta a la intubación en $50 \%$ de los pacientes, Cp aprox. $300 \mathrm{pcg} / \mathrm{mL}$ (bolo inicial a $12 \mu \mathrm{g}$ IV) la cual puede ser recomendada para intubación en pacientes paralizados, pero puede ser insuficiente cuando no se utiliza bloqueador muscular e hipnóticos ${ }^{3}$.

En la fase de mantenimiento la perfusión es manual con una tasa entre $0.0002-0.0006 \mu \mathrm{g} / \mathrm{kg} / \mathrm{min}$ o $0.15-0.22 \mathrm{mg} / \mathrm{kg} / \mathrm{h}$ con una Cp aprox. 0.200-600 pcg, respectivamente. Se debe disminuir la Cp hasta $0.25 \mathrm{ng} / \mathrm{mL}$ para iniciar con ventilación espontánea; cerrar la infusión entre 15 a 20 minutos antes del fi n de la cirugía para obtener un despertar con adecuada analgesia postoperatoria. TCI plasma o sitio efector (modelo de Geps) con dianas de 0.2-0.6 ng/mL mostrando una adecuada estabilidad transanestésica con un suave y confortable despertar en corto tiempo (ventilación espontánea rango entre $0.13-0.03 \mathrm{ng} / \mathrm{mL}$ ).

Utilizando la Anestesia Intravenosa Total (TIVA) o infusión controlada por objetivo (TCI) el efecto del propofol Ce $3 \mu \mathrm{g} / \mathrm{mL}$ y sufentanyl Ce $0.4 \mathrm{ng} / \mathrm{mL}$ proveen una anestesia profunda con recuperación de la conciencia más rápida que con fentanyl o alfentanyl, debido a que es un fármaco que se acumula menos y tiene un mejor perfil para perfusión ${ }^{3,16}$.

EL remifentanilo es un opioide agonista $\mu$, que únicamente puede ser administrado vía IV (glicina, neurotóxica). Su cinética al bolo y perfusión es independiente de la dosis total, y de la duración de la infusión (acumulación mínima en pacientes con severa disfunción hepática o renal). Al suspender la infusión, su efecto se termina en forma rápida (4-8 minutos) requiriendo de analgesia multimodal, desde este momento y complementar con AINES intravenosos, y opioides débiles, para brindar analgesia de transición durante el postoperatorio: tramadol de $1-2 \mathrm{mg} / \mathrm{kg}$ o morfina 100-200 $\mu \mathrm{g} / \mathrm{kg}$, en casos especiales buprenorfina de 2-5 $\mathrm{mg} / \mathrm{kg}$ por dosis y ketamina $0.2 \mathrm{mg} / \mathrm{kg}$ IV para evitar la hiperalgesia inducida por opioides potentes al suspender la perfusión. Inducción: bolo (no recomendado): $1 \mu \mathrm{g} / \mathrm{kg}$ IV alta posibilidad de causar tórax leñoso. Mantenimiento: A) remifentanyl perfusión manual: iniciar perfusión a (Cp aprox. 0.003-0.006 $\mu \mathrm{g} / \mathrm{mL})$, es decir, tasas de 0.12 a $0.24 \mu \mathrm{g} / \mathrm{kg} / \mathrm{min}$ incrementando la perfusión de forma gradual (escalonada en los primeros 3 a 5 minutos). B) Remifentanyl TCI (modelo de Minto) a modo plasma o sitio efecto, con dispositivos como el Rugloop o la Orchestra Base Primea.
Obtener dianas de 3-5 ng/mL, con propofol u halogenados; incluso hasta10 $\mathrm{ng} / \mathrm{mL}$ cuando se realiza TIVA con midazolam ${ }^{3,16}$.

En el salón de cirugía del hospital IESS de Ibarra se utiliza con mayor frecuencia el remifentanilo como primera línea de opioide en la tiroidectomía transoral asociado al sevofluorano al $1 \%$, oxígeno al $0,6 \%$ con inducción de propofol a dosis de $2 \mathrm{mg} /$ $\mathrm{kg}$, relajación muscular con bromuro de rocuronio a dosis de 0,6 $\mathrm{mg} / \mathrm{kg}$ y analgesia multimodal con tramadol, o bupremorfina, complementado con por vía intravenosa con AINES (ketorolaco,matamizol,paracetamol) con buenos resultados y como analgesia de rescate de 50-100 mcg de fentanylo.

\section{Período postoperatorio}

Los cuidados postoperatorios habitualmente son simples. La vigilancia se limita a una laringoscopia postoperatoria con fonación y a un control de la calcemia y de los niveles séricos de hormona paratiroidea (PTH). Los pacientes vuelven a su domicilio días después del postoperatorio, en función de los hábitos de cada centro, del entorno del paciente y de la necesidad de suplementos cálcicos en caso de hipoparatiroidismo postoperatorio. No existe ninguna justificación para la realización de una profilaxis antitrombótica, salvo en el caso de los pacientes que tengan una afección que precise la reinstauración precoz de un tratamiento anticoagulante. El dolor postoperatorio suele ser moderado durante las primeras 12 horas. El mejor tratamiento consiste en la asociación de una ALR (bloqueo cervical superficial) y de analgésicos de nivel $2^{5}$

Los antiinflamatorios no esteroideos disminuyen la intensidad del dolor postoperatorio sin aumentar el riesgo de hemorragia y hematoma de la celda tiroidea ${ }^{18,19}$. También se pueden utilizar los antihiperalgésicos como la gabapentina. Se permite la reanudación de ingesta de líquidos y sólidos en el período inmediatamente posterior ${ }^{5}$.

\section{CONCLUSIONES}

Un eslabón fundamental de la anestesia para tiroidectomía transoral es realizar esta intervención en condiciones eutiroidea del paciente. La preparación preoperatoria está dirigida especialmente a los pacientes hipertiroideos para prevenir el acceso tirotóxico. La técnica anestésica de elección se basa en el uso de anestesia general balanceada, $\mathrm{o}$ anestesia total intravenosa con $\mathrm{o}$ sin insuflación de $\mathrm{CO}_{2}$ y monitorización no invasiva. El equipo quirúrgico, debe, paralelamente a su procedimiento de exéresis, controlar la hemostasia, la función de los nervios recurrentes y de las paratiroides, asegurando una tasa de complicaciones lo más baja posible. De forma general los cuidados anestésicos del paciente sometido a tiroidectomía transoral no difieren de las otras técnicas quirúrgicas mínimamente invasiva del tiroide.

\section{Referencias bibliográficas}

1. Cadena E. Tiroidectomía axilar endoscópica video asistida 3D. Revista Colombiana de Cancerología.(Internet) 2017 Nov.(citado el 20 de Feb.2017); 20(4).190-197.Disponible desde: http://dx.doi. org/10.1016/j.rccan.2016.09.003

2. Granell J. Introducción a la cirugía robótica en cabeza y cuello;(Internet) 2015, Oct. (citado el 01 de Ene.2017) Disponible desde: https://www.researchgate.net/publication/283212117

3. Martínez R. Perfusiones de opioides en cirugía otorrinolaringológica y cirugía de cabeza y cuello. Revista Mexicana de Anestesiología. (Internet) 2015 junio(Citado el 52 de enero 2017).38(250-54).Disponible desde: http://www.medigraphic.org.mx

4. Velásquez S. Manejo perioperatorio del paciente para cirugía e tiroides. Mexicana de Anestesiología.(Internet) 2007 abril-junio,(Citado 20 de enero 2017) 30(185-92).Disponible desde: http://www.medigraphic.com/pdfs/rma/cma-2007/cmas071ae.pdf 
5. Garot M. Anestesia y reanimación en la cirugía de la glándula tiroidea. Elsevier.(Internet). 2015.Nov.(Citado 15 de Ener)41(1-10) Disponible desde: http://dx.doi.org/10.1016/S1280-4703(15)73874-0

6. Bajwa S. Anesthesia and thyroid surgery: The never ending challenges. US National Library of Medicine National Institutes of Health. (Internet) 2013 mraz.(Citado 5 de enero)17(228-34)Disponible desde: http://www.ijem.in/article.asp?issn=2230-8210;year=2013; volume $=17$;issue $=2$; spage $=228$; epage $=234$; aulast $=$ Bajwa

7. Villar J. Vía clínica de tiroidectomía. Elsevier.(internet) 2015 Mayo(Citado 4 de feb);93(283-99).Disponible desde: http://www.elsevier. es/es-revista-cirugia-espanola-36-articulo-via-clinica-tiroidectomia-S0009739X15000159

8. Iglesias JL. NOVEDADES EN VÍA AÉREA DIFÍCIL (Internet): 1era.Ed Editorial Universidad de Salamanca España; 2016.Nov. (Citado 21 de Enero).Disponible desde: https://books.google.es/ books?hl=es\&lr=lang_es|lang_en\&id=LbYgDgAAQBAJ\&oi=fnd\&pg=PP1\&dq=Iglesias+JL.+NOVEDADES+EN+VÍA+

9. O'Dell K. Predictors of difficult intubation and the otolaryngology perioperative consult. Elsevier. (Internet) 2015 Junio(Citado el 25 de Enero 2017) ; 33(2). Disponible desde: https://www.ncbi.nlm. nih.gov/pubmed/25999002

10. Castelao J. Patología mediastínica. Dialnet. (Internet)2014;(Citado 7 de Feb. 2017) 11(67). Disponible desde: https://dialnet.unirioja.es/ servlet/articulo? codigo $=4915036$

11. Puerto J. Bocio endotorácico. Presentación de dos casos. MediSur. (Internet) 2016 Octubre;(Citado el 13 de Enero 2017 14(5).Disponible desde: http://scielo.sld.cu/scielo.php?script=sci_arttext\&pid=S1727-897X2016000500012

12. Rosato L, Carlevato M, De Toma G, Avenia N. Recurrent Laryngeal Nerve Damage and Phonetic Modifications after Total Thyroidectomy: Surgical Malpractice Only or Predictable Sequence? World Journal of Surgery.(Internet) 2005 Junio;(Citado 14 de Feb.) 29(6). Disponible desde: https://www.ncbi.nlm.nih.gov/pubmed/15895296

13. Echternach M, Maurer C, Mencke T, Schilling M, Verse T, Richter B. Laryngeal complications after thyroidectomy: is it always the surgeon? Medline.(Internet) 2009 Febrero;(Citado 28 de Feb 2017) 144(2).Disponible desde: https://www.ncbi.nlm.nih.gov/ pubmed/19221326
14. Farrag T, Samlan R, Lin F, Tufano R. The utility of evaluating true vocal fold motion before thyroid surgery. Medline.(Internet) 2012 Febrero; (Citado 12 de Enero 2017)116(2). Disponible desde: https://www.ncbi.nlm.nih.gov/pubmed/16467711

15. Boussofara M, Raucoules A. Farmacología de las benzodiazepinas utilizadas en anestesia-reanimación. Sparrho.(Internet) 2016 Noviembre(Citado el 21 de Enero 2017)42(4):1-11Disponible desde: http://www.sciencedirect.com/science/article/pii/ S1280470316807227

16. Miller R. Miller Anestesia.(Internet) 8th ed.: España Elsevier; 2016. Marzo (Citado el 15 de Febrero de 2017).Disponible desde: https:// booksdemedicinablog.wordpress.com/2016/03/17/anestesia-demiller-8va-edicion/

17. Tafur L, Serna A, Lema E. Fentanilo PK/PD, un medicamento vigente. Colomb Anesteiol.(Internet) 2010 Abril; (Citado el 3 de Feb.de 2017)38(1).Disponible desde: http://www.revcolanest. com.co/es/fentanilo-pk-pd-un-medicamento-vigente/articulo/ S0120334710810056/

18. N Akihiro ,A Hideo. Una nueva técnica transoral de la tiroidectomía endoscópica con enfoque premandible sin gas. Total Endoscopic Thyroidectomy.(Internet) 2013 Abril,(Citado el 8 de Enero 2017)27(4) P 1105-1110 Disponible desde:https://link.springer. com/article/10.1007/s00464-012-2588-6

19. Casanova D. Cirugía mínimamente invasiva de tiroides y paratiroides; Scielo analylic (Internet) 2012.(Citado de Enero 2017) 28(3) Disponible desde http://scielo.isciii.es/scielo.php?script=sci_arttext\&pid=S1137-66272005000600012

20. Fourcade O, Sanchez P, Kern D. Propacetamol and ketoprofen after thyroidectomy. European Society of Anaesthesiology.(Internet) 2012 Mayo;(Citado el 20 de febrero) 22(5).Disponible desde: https:// doi.org/10.1017/S0265021505000645

Recibido: Marzo de 2017.

Aprobado: Abril de 2017. 